\title{
THEIA: an advanced optical neutrino detector
}

\section{Stephane Zsoldos ${ }^{a, b, *}$}

${ }^{a}$ University of California, Berkeley, Department of Physics, CA 94720, Berkeley, USA

${ }^{b}$ Lawrence Berkeley National Laboratory, 1 Cyclotron Road, Berkeley, CA 94720-8153, USA

E-mail: zsoldos@berkeley.edu

THEIA is a proposed large-scale novel neutrino detector, designed with the ability to discriminate between Cherenkov and scintillation signals. The design consists of a cylindrical tank viewed by inward-looking PMTs and filled with a novel target, such as water-based liquid scintillator (WbLS) or other scintillators, which would allow simultaneous reconstruction of particle direction from the Cherenkov signal, with the energy resolution and low threshold of a scintillator detector. THEIA would have a broad physics program ranging from low energy solar to high energy accelerator neutrinos.

*** The European Physical Society Conference on High Energy Physics (EPS-HEP2021), ***

*** 26-30 July $2021 * * *$

*** Online conference, jointly organized by Universität Hamburg and the research center DESY ***

${ }^{*}$ Speaker 


\section{Introduction}

Historically, neutrino experiments have designed either Liquid Scintillator (LS) or WaterCherenkov (WC) detectors to detect the incoming leptons from neutrino interactions inside huge volumes. LS would suit best low-energy neutrino detection, for example, MeV neutrinos from solar or artificial sources like nuclear reactors, thanks to the high light yield output by the scintillation process. But while WC detectors can not access physics below the Cherenkov threshold, they offer $O(100 \mathrm{~m})$ attenuation length allowing the construction of mega-tonne scale detectors. Moreover, the signal topologies inside WC allow the original neutrino direction reconstruction, compared to the isotropic light production by scintillation. THEIA [1] is a proposed detector that reconciles both technologies, gathering the advantages of a high output light yield without losing the direction information of the incoming particle, providing particle ID at high energies and background discrimination at low-energy. Therefore, THEIA could have a broad physics program in nuclear, high-energy, and astrophysics, ranging from hundreds of $\mathrm{keV}$ to $\mathrm{GeV}$, including the observation of low- and high-energy solar neutrinos, determination of neutrino mass ordering and the CP-violating phase $\delta_{\mathrm{CP}}$, observation of the diffuse supernova neutrino background and supernova neutrino burst, measurement of the geoneutrino flux, sensitive searches for nucleon decay and, ultimately, a search of neutrinoless double-beta decay. In the following section, we will describe the cutting edge developments in the target material and photodetection R\&D leading to THEIA, and the sensitivities to study fundamental neutrino properties and astrophysical objects. For the latter two main scenarios are explored, one in which THEIA would reside in a cavern the size and shape of those intended to be excavated for the Deep Underground Neutrino Experiment (DUNE), which we call TheIA-25, and a larger 100-kt version (THEIA-100) that could achieve an even broader and more sensitive scientific program.

\section{Detector R\&D}

In both scenarios, THEIA geometry consists of a cylindrical tank, and facing inwards arranged against the inner wall of the cylinder will be hundreds of thousands of photo-detectors capable of detecting the faint signatures of neutrinos interacting within. THEIA scientific program benefits in many cases from the detector's ability to discriminate between the scintillation and Cherenkov photons. This can be achieved in several ways, and ultimately THEIA would want to combine all techniques available at the same time.

\subsection{Water-based liquid scintillator}

THEIA's physics goals sensitivity increases with respect to the target volume at disposal. Choosing the suitable material to fill the detector must therefore have a large attenuation length while being safe and easily manipulable at a large scale, comparable to WC detectors, for example. Developed at BNL, Water-based Liquid Scintillator (WbLS) is a cocktail of pure water and oil-based liquid scintillator [2]. Since oil and water are not miscible, an additional component called surfactant is introduced to hold the scintillator molecules in water in a micelle structure. By introducing a small amount, typically $3 \%$ of liquid scintillator into the water, the cocktail light yield can eventually be adjusted to allow detection of particles below Cherenkov threshold while not sacrificing the 
directional capability, cost, or environmental friendliness. This target material is the leading candidate for THEIA, and it is actively characterized by the collaboration to precisely measure its light yield, emission spectrum, scintillation time profile and both scattering and attenuation length [8] [9] [10] [11] [12].

\subsection{New generation photodetectors}

The separation of scintillation from Cherenkov photons is based on the physical process underlying their production. While scintillation is an isotropic process, Cherenkov light is emitted in a cone forward the direction of the charged particle. Cherenkov light is produced much more quickly than scintillation, and on a continuous $1 / \lambda$ wavelength spectrum, compared to a peak for scintillation at $O(400 \mathrm{~nm})$. Maximizing the amount of information from the photons collection, THEIA's photodetector system must achieve the fastest time resolution to sort scintillation from Cherenkov photons by their arrival, and be sensitive to their wavelength. Very fast photo-multipliers (PMTs) can nowadays achieve a ns transit time spread (TTS) resolution and at a large photocathode size. Moreover, the recent development of large-area red-sensitive PMTs could offer the same fast TTS and sensitivity to the photons' larger wavelength. Large area picosecond photo-detectors (LAPPDs) [3] are $20 \mathrm{~cm} \times 20 \mathrm{~cm}$ imaging photosensors with single photoelectron time resolution below $100 \mathrm{ps}$ and sub-cm spatial resolutions. Such design allows LAPPDs to offer a significant advantage over conventional PMTs. While PMTs are single-pixel detectors, LAPPDs can be considered imaging detectors able to reconstruct light patterns, such as Cherenkov rings, in both time and position. Their spatial resolution allows precise neutrino interaction vertex reconstruction and separation of multiples rings originating from the same event, such as pion-induced tracks.

\subsection{Dichroic filters}

In addition to several advances in photodetectors $\mathrm{R} \& \mathrm{D}$, the THEIA collaboration is looking beyond the photosensors themselves and considering optics for using photodetectors more efficiently. Traditional light collectors such as Winston Cones but using specular reflection off mirrors could also enhance coverage. One up-and-coming optical concept is the application of dichroic filters to separate light by wavelength [4] [13]. This would provide a strong additional handle for discriminating between the largely monochromatic scintillation light and broad-band Cherenkov light, as well as enabling better correction for chromatic dispersion of Cherenkov photons.

\section{Physics sensitivities}

THEIA program offers a significant advantage thanks to the nature of WbLS, which allows it to be tunable in terms of liquid scintillator content. Therefore THEIA can adopt a phased program to address the science priorities. In the following sections, three experimental stages are described: during the first, the performance of THEIA as a long-baseline neutrino experiment is shown; the second section discusses the sensitivity to low-energy (anti)neutrinos; the third presents the prospects for the search of neutrinoless $\beta \beta$-decay in THEIA. A recent white paper has been released presenting this approach [1]. The detector is assumed to be installed at the Sanford Underground Research Facility (SURF, 4300 m.w.e.) and two different configurations are considered: a baseline option with a $25 \mathrm{kt}(17 \mathrm{kt} \mathrm{FV})$ detector that will fit a fourth DUNE 
cavern, known as "module of opportunity", and an ideal configuration foreseeing a $100 \mathrm{kt}(70 \mathrm{kt}$ FV) experiment.

\subsection{Phase I: high-energy program}

Starting with the lowest fraction of liquid scintillator inside the WbLS cocktail, around [1$3] \%$, THEIA-25 can complement the DUNE detectors exploiting the LBNF neutrino beam $(1300 \mathrm{~km}$ baseline) to provide a measurement of neutrino oscillation parameters obtained with a detector affected by different systematics, thus strengthening the oscillation results on the LBNF beam. The sensitivity to mass hierarchy and to $\delta_{\mathrm{CP}}$ has been studied using the GLoBES framework [5] under the conservative assumption that THEIA will reproduce the detector performance of current WCD. The results of the analysis, obtained considering 9 samples of events divided for different topology, are presented in Fig. 1 and show that in the baseline configuration THEIA-25 has a sensitivity compatible with a single DUNE module.
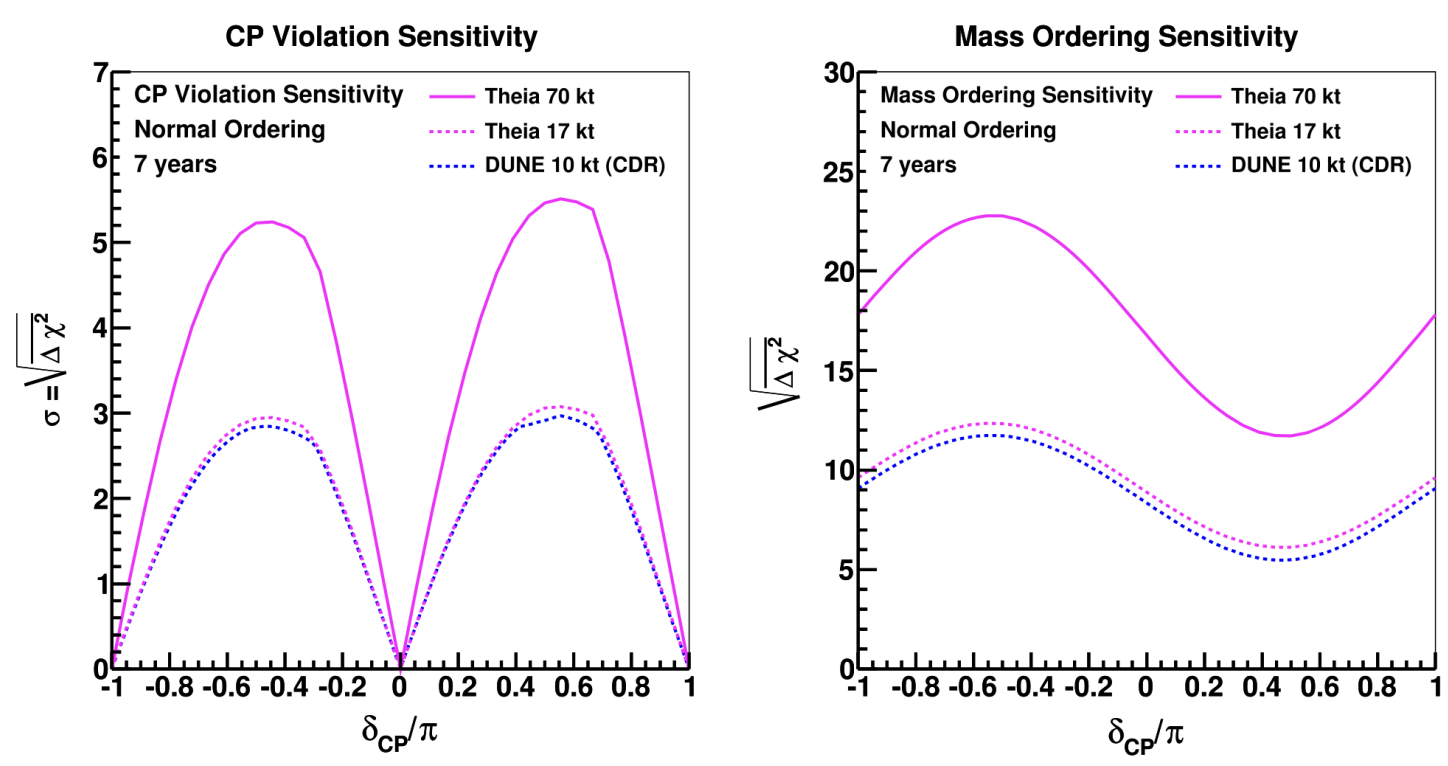

Figure 1: Sensitivity to CP violation (left) and sensitivity to determination of the neutrino mass ordering (right), as a function of the true value of $\delta_{C P}$, for the Theia 70-kt fiducial volume detector (pink). Also shown are sensitivity curves for a 10-kt (fiducial) LArTPC (blue dashed) compared to a 17-kt (fiducial) THEIA-25 WCD detector (pink dashed). Seven years of exposure to the LBNF beam with equal running in neutrino and antineutrinomode is assumed.

During this phase Theia can also achieve a new measurement of the ${ }^{8} \mathrm{~B}$ flux from the sun, and more astrophysical results. For supernova burst neutrinos, the high statistics $(\simeq 23000$ events for a $10 \mathrm{kpc}$ ) and low-threshold, flavor-resolved neutrino spectra can achieve a few degrees resolution inside Theis-100 [1]. At LBNF, the combination of WbLS from TheiA-25 and LAr from DUNE detectors at the same site allows for the co-detection of neutrinos and antineutrinos.

The diffuse supernova neutrino background, or the isotropic flux of neutrino from all supernova explosions in the Universe, could be detected at $5 \sigma$ in 8 years inside THEIA-25 or only 2 years in THEIA-100 [1]. 


\subsection{Phase II: low-energy program}

Increasing the fraction of LS inside the cocktail towards [5-10]\%, the detection threshold inside Theia will lower further down to include more solar neutrino physics. Specifically, the search for new physics by the measurement of the ${ }^{8} \mathrm{~B}$ MSW transition or spectrum up-turn, which is a matter enhanced flavor conversion around [2-7] MeV. Another goal would be to perform precision measurement of the Carbon-Nitrogen-Oxygen cycle neutrino flux recently observed by the Borexino collaboration, which is achievable in THEIA thanks to the directional information reconstruction that will provide precious information about the Sun metallicity [6].

THEIA would also detect antineutrinos from the inverse-beta decay (IBD) interaction, especially from geo- and reactor sources. THEIA-25 can detect ${ }^{235} \mathrm{U}$ and ${ }^{232} \mathrm{Th} \bar{v}_{e}$ emitted from Earth's crust and mantle layers. At SURF THEIA-25 would explore a new geographical location compared to previous experiment which detected geoneutrinos (Borexino, KamLAND). Preliminary studies suggest that THEIA-25 could detect the geoneutrino flux at $5 \sigma$ in only 90 days, and after a year provide a measurement of the U/Th contribution ratio, and also on the crust and mantle flux.

THEIA-25 could also be sensitive to antineutrinos from the nuclear plants in the US and Canada, at a baseline around $O(1000 \mathrm{~km})$, allowing a demonstration of long-range reactor monitoring, and potentially some sensitivity to solar oscillation parameters.
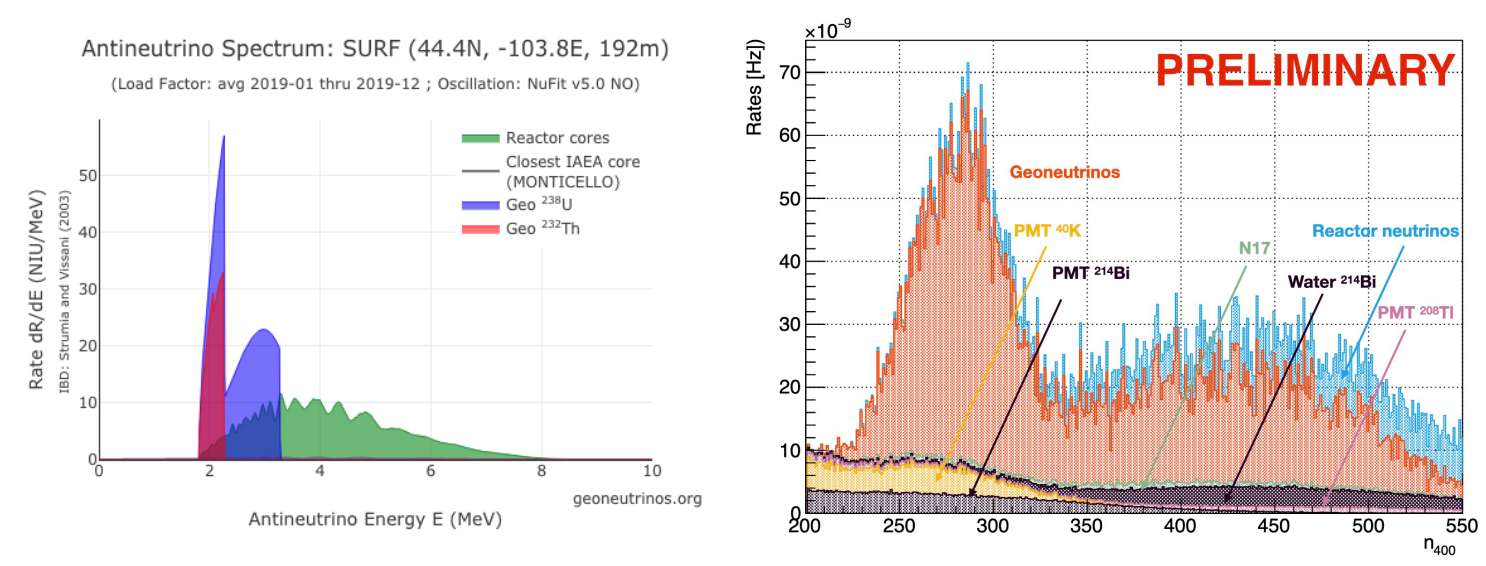

Figure 2: Left: The expected antineutrino flux at SURF [7]. Right: PDFs of signal and backgrounds expected in THEIA-25, in terms of a the number of hits in a $400 \mathrm{~ns}$ after the trigger.

\subsection{Phase-III: Neutrinoless- $\beta \beta$ decay}

This last and most ambitious phase of the THEIA project consists of loading a $0 v \beta \beta$ candidate isotope dissolved into $8 \mathrm{~m}$ radius nylon balloon filled with ultra-pure LS and immersed in the target volume that shields it from environmental radioactive background. ${ }^{130} \mathrm{Te}$ is considered a very promising option for the search of $0 v \beta \beta$ in THEIA given its large natural abundance and the solubility in LS already demonstrated by the SNO+ collaboration. The sensitivity to a $0 v \beta \beta$ signal depends crucially on the exposure, energy resolution and background level of the experiment. The energy resolution of the detector has been assumed to be $\simeq 3 \% / \sqrt{E}$ that is achievable using a cocktail of $\mathrm{LAB}+2 \mathrm{~g} \mathrm{~L}^{-1} \mathrm{PPO}$ and a very high photocoverage of $90 \%$. Radioactive contamination 
in the LS and in the nylon vessel as well as cosmogenic background are assumed to be under control thanks to advanced purification procedures, volume fiducialization and tagging techniques based on events topology and space-time correlation. Provided these experimental conditions are met and given its very large mass, with a 10 years exposure THeiA will be competitive with proposed beyond-ton-scale searches for $0 v \beta \beta$ decay.

\section{Conclusion}

Progress in the novel target materials and photodetector technologies opened the path for the next-generation neutrinos experiments, and THEIA will employ the advantages of these developments to achieve: low energy threshold, good energy and position resolutions, directionality, large exposure and to tackle a broad physics agenda: neutrino oscillations, solar, supernova neutrinos, and $0 v \beta \beta$ decay.

\section{References}

[1] M. Askins et al. [Theia], Eur. Phys. J. C 80 (2020) no.5, 416

[2] M. Yeh, S. Hans, W. Beriguete, R. Rosero, L. Hu, R. L. Hahn, M. V. Diwan, D. E. Jaffe, S. H. Kettell and L. Littenberg, Nucl. Instrum. Meth. A 660 (2011), 51-56

[3] B. W. Adams et al. [LAPPD], [arXiv:1603.01843 [physics.ins-det]].

[4] T. Kaptanoglu, M. Luo and J. Klein, JINST 14 (2019) no.05, T05001

[5] P. Huber, J. Kopp, M. Lindner, M. Rolinec and W. Winter, Comput. Phys. Commun. 177 (2007), 432-438

[6] B. J. Land, Z. Bagdasarian, J. Caravaca, M. Smiley, M. Yeh and G. D. Orebi Gann, Phys. Rev. D 103 (2021) no.5, 052004

[7] S. Dye and A. Barna, [arXiv:1510.05633 [physics.ins-det]].

[8] J. Caravaca, F. B. Descamps, B. J. Land, J. Wallig, M. Yeh, and G. D. Orebi Gann, Phys. Rev. C95 055801 (2017).

[9] J. Caravaca, F. B. Descamps, B. J. Land, M. Yeh, and G. D. Orebi Gann, Eur. Phys. Jour. C $77: 811$ (2017).

[10] J. Caravaca, B. J. Land, M. Yeh, and G. D. Orebi Gann, Eur. Phys. Jour. C 80 : 867 (2020).

[11] D. R. Onken, F. Moretti, J. Caravaca, M. Yeh, G. D. Orebi Gann, E. D. Bourret, Mat. Adv. 1 : 71-76 (2020)

[12] T. Kaptanoglu, E. J. Callaghan, G. D. Orebi Gann, arXiv:2110.13222 (submitted to Eur. Phys. Jour. C)

[13] T. Kaptanoglu, M. Luo, B. Land, A. Bacon, J. R. Klein, Phys. Rev. D 101 : 072002 (2020) 